\title{
Foscolo e Locke
}

Foscolo et Locke

Foscolo and Locke

\section{Matteo Palumbo}

\section{CpenEdition}

\section{Journals}

Edizione digitale

URL: http://journals.openedition.org/cei/2341

DOI: $10.4000 /$ cei.2341

ISSN: 2260-779X

Editore

UGA Éditions/Université Grenoble Alpes

\section{Edizione cartacea}

Data di pubblicazione: 30 juin 2015

Paginazione: 49-63

ISBN: 978-2-84310-289-9

ISSN: 1770-9571

Notizia bibliografica digitale

Matteo Palumbo, «Foscolo e Locke», Cahiers d'études italiennes [Online], 20 | 2015, online dal 01 janvier 2017, consultato il 26 mars 2021. URL: http://journals.openedition.org/cei/2341; DOI: https://doi.org/ 10.4000/cei.2341 


\title{
FOSCOLO E LOCKE
}

\author{
Matteo Palumbo \\ Università di Napoli Federico II
}

I. Nel suo bel libro su Foscolo e la letteratura inglese, Sandra Parmegiani ha dedicato un capitolo intero ai rapporti tra Foscolo e Locke, ricostruendo le tracce lasciate dal filosofo inglese nella riflessione e nella cultura di Foscolo $^{\mathrm{I}}$. Da questo saggio è necessario prendere le mosse, provando ad aggiungere qualche considerazione supplementare intorno al significato che questa presenza suggerisce.

Orienterò il mio intervento lungo tre assi. Il primo percorso connette la presenza di Locke all'interno di una costellazione teorica più grande, con cui fa sistema. La fusione tra autori di specie diversa contribuisce, in maniera essenziale, a definire il nucleo delle idee di Foscolo. Il secondo aspetto riguarda l'utilizzazione di Locke in relazione a un tema a cui Foscolo è legato in maniera quasi programmatica: il ruolo del poeta in mezzo alla contemporaneità ostile e l'estraneità morale e artistica ai disvalori che la governano. La terza linea tocca alcune questioni connesse più direttamente alla téchne poetica, cioè alla forma specifica del fare poesia e ai mezzi che la regolano. I tre aspetti sono naturalmente intrecciati e compongono le facce di un unico interesse. Convergono insieme nel riconoscere nel filosofo empirista uno dei pensatori a cui Foscolo rivolge un tributo costante e ammirato.

2. Come è ben noto, il primo indizio di un'ammirazione esplicita per l'autore del Saggio sull'intelletto umano si trova nel Piano di Studj, dove Locke compare insieme con Yves-Marie André nella casella della Metafisica, qualificata dalla dizione di «Entusiasmo d'anima». Va altresì ricordata la nota successiva, che sottomette tutti i riferimenti della morale, della politica,

I. S. Parmegiani, Ugo Foscolo and English Culture, London, Legenda, $201 \mathrm{I}$. 
della metafisica e della teologia a Bacone, «di cui tutte l'opere sono la chiave universale d'ogni filosofia» ${ }^{2}$. La conoscenza di Locke, o almeno l'inserimento del suo nome nel canone privato che il giovane Foscolo sta allestendo, è il segno di una sintonia immediata, affidata per ora a formule generiche. Merita, tuttavia, attenzione un minimo indizio. Il richiamo ad André e la dizione "entusiasmo d'anima», associata da Foscolo in una celebre definizione al campo della lirica ${ }^{3}$ e «contrassegno di una poesia che anela al soprannaturale e divino" ${ }^{4}$, lascerebbero pensare a un interesse letterario più che filosofico: o, almeno, al privilegio accordato a fattori di stile oltre che di pensiero.

Le ragioni di questa possibilità si spiegano meglio con i riferimenti offerti da successive testimonianze. Tali ulteriori riscontri definiscono in termini più precisi i motivi di un'adesione reiterata all'esperienza lockiana. Essa esprime un paradigma intellettuale, che obbedisce ad alcuni presupposti esemplari. Si nutre della potenza delle idee; riveste l'energia che esse contengono con parole idonee; mostra indifferenza alla malevolenza di detrattori interessati e mediocri. Una figura intellettuale così elaborata mantiene fedeltà al mandato di verità che costituisce il suo obiettivo, e, in cambio della costanza verso i propri principi, ottiene di durare nel tempo, oltre il ciclo limitato e finito della vita del corpo. Il caso di Locke si trasforma così in un emblema. Alla novità delle sue idee aggiunge l'efficacia dello stile e la dignità del comportamento: tre qualità che descrivono, agli occhi di Foscolo, la figura dell'artista come avanguardia della società.

Il richiamo alla biografia di Locke appare nella lezione seconda Della morale letteraria e riguarda tutti i molteplici aspetti prima indicati. Essi comprendono tanto l'originalità dell'elaborazione teorica quanto i modi di trasmetterla. Vita e opera appaiono combinati e diventano ammirevoli proprio attraverso la ricchezza del loro reciproco integrarsi.

La novità del pensiero lockiano è certo indiscussa. Essa si inscrive dentro la legge che governa il movimento fatale delle filosofie. La logica che indirizza le idee umane prevede, infatti, un'incessante dialettica, che avvia un movimento permanente. Questa dinamica, a cui Foscolo collega una riconoscibile e ovvia formula vichiana, mette in moto il «corso e ricorso perpetuo di molti errori e di pochissime verità, di insufficienti esperienze e

\footnotetext{
2. EN VI, p. 3.

3. EN VII, p. 326: «la poesia lirica canta con entusiasmo le lodi de' numi e degli eroi».

4. E. Neppi, Foscolo pensatore europeo. La dualità dell'essere nell'"Orazione pavese", in U. Foscolo, Dell'origine e dell'ufficio della letteratura, a cura di E. Neppi, Firenze, Olschki, 2005, p. 22.
} 
d'ipotesi immaginarie che pur giovano all'intento della natura, che sembra essere di tener sempre in moto le passioni e l'ingegno di tutti i viventi»'s. L'evoluzione da una tesi affermata a una nuova congettura è inarrestabile. Obbedisce alla mutevolezza delle passioni e dei loro obiettivi ${ }^{6}$.

E, tuttavia, nel perpetuo alternarsi di un sistema al posto di un altro, c'è chi resiste e guadagna una vita più lunga. Il Foscolo difensore della grandezza e del valore dell'arte prevede naturalmente che le parole della poesia, come aveva sostenuto con forza qualche anno prima nei Sepolcri, possano donare una vita più lunga: alternativa alle leggi della natura e libera dai suoi vincoli. Questa vita seconda, posta al di là della morte, si identifica pienamente con la dignità dell'uomo di lettere. Il suo lavoro conosce una «certezza morale», che immunizza dall' «effetto micidiale» di dimenticanza, che minerebbe la sostanza stessa della vita di un artista:

Ma io voglio omai accordare ciò che sarebbe d'effetto micidiale alle lettere ove non s'accordasse; ed è, che il letterato abbia non solo lusinga ma piena certezza morale che, quand'egli scriva con eloquenza e con verità, il suo nome volerà chiaro ed eterno per le bocche degli uomini; alla quale certezza aggiungeremo che egli sia sì fattamente innamorato della gloria, che la scorga in tutta la sua bellezza, e che con la fantasia degli innamorati le ascriva un non so che di divino per cui egli, accompagnato da questa divinità della gloria, possa superare la morte e vivere oltre il sepolcro7 .

I temi forti della tradizione letteraria umanistica si affacciano con tutta la loro massiccia evidenza. Gloria ed eternità, frutto dell'amore per la verità tradotta in eloquenza, sono il premio e la ricompensa dell'intellettuale libero, animato unicamente dall'impegno a parlare in nome della comunità intera degli uomini. Questo premio morale non libera, tuttavia, da pene spiacevoli né garantisce un'esistenza serena. Il nome e il caso «memorable» (come Foscolo stesso lo etichetta) di Locke appaiono precisamente a illustrare questa tesi. L'esperienza del filosofo evoca il modello dell'uomo di lettere designato a vivere nel tempo più lungo della storia e abilitato a prolungare la sopravvivenza dei suoi pensieri al di là di ogni limite, tracciato dalla moda o semplicemente imposto dall'usura.

Il primo atto encomiastico che Foscolo compie sancisce perentoriamente il peso e il ruolo che il filosofo occupa nello spazio della filosofia moderna:

5. EN VII, p. I26.

6. «E quanto alle scienze, il caso sovente e sempre l'opinione degli uomini fanno sottentrare nuove opinioni e nuovi sistemi che atterrano i precedenti; onde tale filosofo che fu reputato al suo tempo sommo interprete della natura fu nell'età che seguì o malignamente, ma vittoriosamente calunniato e deriso, o giustamente impugnato da' promotori d'altri sistemi, i quali, come tutte le cose terrene, dovranno essere un giorno combattuti e vinti e obbliati» (ibid.).

7. Ibid. 
Giovanni Locke per universale consenso arricchì il suo secolo del libro più eloquente e più utile fra quanti mai illuminarono il mondo; più eloquente, perchè non solo è scritto con tutta schiettezza di lingua e vigore di stile e calore di pensiero, che è reputato in ciò esemplare da tutti gli Inglesi, ma ben anche perchè è disegnato con mirabile architettura di parti, eseguito con profondità di ragionamento, e dotato di quel tocco magico della persuasione a cui il solo stile e il solo ragionamento non giungono, ma che nasce da un certo vigore di concepire le idee e da certo amore nell'esporle; doti che dagli antichi Greci e Latini erano credute doni celesti, onde consacrarono templi ed altari alla dea della Persuasione ${ }^{8}$.

I predicati che Foscolo sceglie sono tra i più cari della sua riflessione estetica. I meriti che fanno del libro di Locke un'opera importante non si restringono solo nell'illuminazione delle menti, liberate dagli errori di sostanza e di metodo. Anche Leopardi, nello Zibaldone, avrebbe sostenuto che il pregio dei libri utili «non consiste per lo più nel porre, ma nel togliere, o dagl'intelletti o dalla vita» ${ }^{9}$ errori e abusi e, per illustrare questa tesi, ricorre precisamente a Locke.

Per Foscolo, oltre al metodo, contano altri fattori. Sono, per esempio, il «calore di pensiero» (basti richiamare l'opposizione calore-fiamma fissata nella Notizia bibliografica) ${ }^{\text {Io }}$, "l'architettura di parti» (che si connette alla «tessitura» dei Sepolcri, difesa con forza nella Lettera a Monsieur Guillon) ${ }^{\text {II }}$, e, infine, la "persuasione» e "l'eloquenza», che compongono un binomio inseparabile, garante della potenza espressiva della parola letteraria e contrassegno della sua utilità sociale ${ }^{\mathrm{12}}$.

Proprio nella Lezione prima su la letteratura e lingua, che appartiene al medesimo contesto speculativo, si può leggere:

L'uso della parola si rende utile, rendendolo grato alle passioni e convincente alle opinioni. Si rende grato alle passioni esercitandole, perchè le passioni non si spengono mai. Si convincono le opinioni dimostrandone il danno e l'utilità. Questa alleanza di passioni e di ragione per mezzo della parola costituisce la persuasione; la persuasione costituisce l'unico fine dell'eloquenza. La poesia, la storia e la facoltà oratoria, che costituiscono la letteratura d'ogni nazione, non cangiano se non le apparenze, perchè tutte stanno nell'eloquenza ${ }^{\mathrm{I} 3}$.

8. Ivi, pp. I26-I27.

9. G. Leopardi, Zibaldone, in Id., Tutte le opere, a cura di W. Binni e E. Ghidetti, Firenze, Sansoni, I993, p. 2707.

IO. «E vuolsi distinguere calore da fiamma; il primo è dote di molti antichi scrittori, e di tutti i primitivi come la Bibbia ed Omero; e la seconda è dote moderna, per lo più francese, specialmente in questi ultimi anni» (EN IV, pp. 493-494).

II. «Ella vede dalle mie note quanto ha sbagliato su' passi da lei citati; molto più su la tessitura la quale dipende dalle transizioni» (EN VI, p. 508).

I2. «Evidenza di lingua, calore ed eleganza» sono richiamate nel Ragguaglio d'un'adunanza de' Pitagorici (EN VII, p. 253).

I3. EN VII, p. 63. 
Resta il fatto che il libro di Locke ha operato una vera rivoluzione rispetto alle opinioni dominanti, combattendo errori e mistificazioni. Leopardi, nello Zibaldone inserisce, a sua volta, Locke nella famiglia di quei pensatori "che hanno veramente mutato faccia alla filosofia» ${ }^{\text {4 }}$. Per Foscolo, il Saggio sull'intelletto umano ha svelato con più radicalità gli inganni della metafisica, da quella platonica a quella cartesiana, sostituendo all'ontologia dell'essere umano la storia delle sue relazioni e dei suoi comportamenti sociali:

Alla bellezza del libro di Locke aggiungesi, come s'è detto, il merito dell'utilità, non tanto per le verità ch'egli espose, quanto per gli errori che dileguò. — E infatti la metafisica platonica e cartesiana, che ingombravano di tanti paradossi la strada delle scienze ne' secoli antichi e moderni, e il gergo delle scienze scolastiche e delle cattedre superstiziose de' claustrali si dileguarono appena pubblicato quel libro; e chi volesse esaminare i sistemi d'Elvezio, di Rousseau, di Bonnet ${ }^{15}$ e d'altri d'ogni nazione sino a Kant che tornò all'idealismo, s'accorgerebbe che, se gli errori sono di questi autori, il fondo della verità de' loro libri è tutto desunto dalle teorie del libro di Locke ${ }^{16}$.

I due opposti paradigmi (uomo di natura e uomo sociale) si fronteggiano come entità astratte. Costituiscono due proiezioni unicamente congetturali. Il primo modello evoca uno stato di natura ipotetico e virtuale, che ha il contrassegno di una costruzione unicamente logica; il secondo richiama, in modo analogo, una distinzione fittizia, "quasi che la società non fosse emanazione necessaria della natura, e l'uomo non fosse animale naturalmente sociale, naturalmente distruttore» ${ }^{17}$. Più giusto, per Foscolo, sostenere, alla luce di Hobbes, "che la natura e la società del genere umano erano una cosa sola ed identica» ${ }^{18}$. Come afferma esplicitamente indirizzandosi al lettore nella prima delle Lettere scritte dall'Inghilterra, Foscolo

I4. G. Leopardi, Zibaldone, cit., p. I857.

15. La stessa tesi è espressa nel Ragguaglio: «È vero che alcuni nelle nostre università si giovano come possono e sanno de' principj di Locke. È vero che il padre Soave tradusse, con le dovute note cattoliche, il compendio che il dott. Wind fece dell'originale ad uso delle donne e de' giovani inglesi. È vero che conosciamo in Italia i sistemi che Rousseau, Elvezio, Condillac, Bonnet ec. desunsero da quell'autore. Ma i sommari, i compendi e i sistemi sono pur sempre ruscelli, canali, torrenti e non mai quel largo, pieno, maestoso, limpido fiume di verità originale. Ė dunque vero che noi abbiamo bisogno d'una versione che non s'è ancora ottenuta» (EN VII, p. 253).

16. EN VII, p. I27.

I7. EN VI, p. 6I5.

I8. «Tutte le alte massime de' platonici sul gius delle genti, ridotte a' minimi termini, appariranno sempre incoerenti. I filosofi distinsero i diritti e i doveri di natura da' diritti e da' doveri di società; quasi che la società non fosse emanazione necessaria della natura, e l'uomo non fosse animale naturalmente sociale, naturalmente distruttore. Tutti gli eloquenti paradossi di Giangiacomo Rousseau derivano da questa fantastica distinzione; tutte le temute verità di Tommaso Hobbes derivano invece dall'avere egli conosciuto che la natura e la società del genere umano erano una cosa sola ed identica. Ma applicando la storia d'ogni gente e d'ogni età all'assioma di Hobbes ed ai corollari di Macchiavelli e di Montesquieu, si ricaveranno i veri e soli diritti della guerra che Ugo Grozio desunse dai fatti» (ibid.). 
invita a osservare "quell'uomo della natura che troverai nella tua famiglia e nella tua città» ${ }^{19}$.

Il nome di Locke è inseparabile da questa prospettiva teorica, che combatte le idee innate o contrasta l'ipostasi di una natura irenica. Egli indica l'alternativa filosofica ai pensieri di Rousseau e ne azzera gli assunti. Le conclusioni implicite di questa rivoluzione antropologica trovano la loro ricapitolazione perfino in forma di tesi:

Da questo terzo capo emerge: $\mathrm{I}^{\circ}$ che lo stato di natura, di guerra e di società sono una cosa sola ed identica, e che non vi possono essere uomini senza una specie qualunque di letteratura; $2^{\circ}$ che le distinzioni di stato di natura e di società sono fantasmi platonici da lasciarsi a Rousseau ed a' suoi partigiani; poichè Rousseau, dividendo la natura dell'uomo dalla società, pianta per principio delle sue declamazioni che le lettere, essendo frutto della società, corrompono la natura dell'uomo ${ }^{20}$.

Nella Considerazione quarta dell'editore sopra le opere di Montecuccoli la liquidazione di qualunque paradigma ideale è perfino sprezzante. Dei filosofi che lo rappresentano più niente è destinato a sopravvivere. Le loro costruzioni vacillano simili a castelli di carta e affondano nel nulla, come sogni inutili e dannosi: «all'aprirsi della storia svaniranno i diritti che Platone, Cicerone, Rousseau e mill'altri attinsero nell'oceano del mondo ideale» ${ }^{2 \mathrm{I}}$.

In una lettera del 3I agosto del I8I4 Foscolo ritorna sul medesimo concetto e lo ribadisce in una forma seccamente alternativa. Da un lato si colloca Locke, con il metodo di cui è difensore; dall'altro lato, invece, resistono i filosofi tedeschi, bloccati nella caparbia difesa delle teorie di perfettibilità e di progresso. Tra i due modelli non c'è nessuna esitazione. Il primo conduce al mondo della storia e della vita; l'altro sfocia nell'universo delle invenzioni irreali:

Locke ha detto: «Figliuoli miei, esaminate i fatti, e troverete i principj; o se non altro dalla serie costante e perpetua di molti fatti imparerete come dovrete condurvi»: - e questi tedeschi dicono: «Dai principj derivano necessariamente i fatti; dunque cerchiamo per la più corta i principj; e i principj sono che l'uomo deve un giorno o l'altro diventare perfetto»; ma cercando la strada da un punto ignoto per arrivare a un punto ancora più ignoto i buoni tedeschi si vanno perdendo, empiono libri di sogni, e non s'intendono neppure fra loro, benché si lodino, e si diffendano ${ }^{22}$.

19. EN V, p. 24I.

20. EN VII, p. 63.

2I. EN VI, p. 6is.

22. Ep. V, p. 228 (lettera alla contessa d'Albany, 3I agosto I8I4). 
Dall'osservazione dei fatti ai principi: è questo il principio della filosofia di Locke, che condanna ogni altra metodologia a partorire solo «libri di sogni».

3. La rivoluzione delle idee non è, tuttavia, l'unica ragione della grandezza di Locke. L'originalità del pensiero si fonde con l'energia dello stile. Agisce con efficacia perché trova uno strumento idoneo nell'articolazione delle parole e delle frasi, senza la cui concretezza i risultati sarebbero assai diversi. La "forza dell'eloquenza» dona un'autorità che mette al riparo. Protegge perfino, finché può, dall'aggressione dei nemici: «Le prove di questo libro erano sì evidenti, e tale la forza dell'eloquenza con cui vennero esposte, che per i primi dieci anni niuno osò turbare nè la pace nè la fama di quell'autore» ${ }^{23}$.

La tregua, però, dura poco. L'azione rivoluzionaria del pensiero è direttamente proporzionale all'ostilità che incontra e che non perdona. Inattaccabile sul piano delle idee, Locke diventa un migliore bersaglio sul piano della vita privata. Saldando l'audacia delle tesi con l'avversione dei letterati e pensatori rivali, Foscolo trasforma il filosofo in un doppio del proprio io ${ }^{24}$. Lo presenta ostaggio della maldicenza, dell'invidia e, insieme, lo mostra indifferente all'arroganza, invulnerabile ai colpi che essa scaglia. Per Foscolo, Locke rientra, in questo modo, nella costellazione dei grandi perseguitati ed esiliati, secondo un modello che da Dante arriverà a se stesso:

Ma poichè s'accorsero che quelle verità non si ristavano nella sola teoria, ma s'erano rivolte alla pratica, primi fra tutti gli ecclesiastici inglesi, e quindi i maestri e discepoli di sistemi ideali si sfrenarono sì dirottamente sul libro, e dal libro, che era per sè stesso insensibile, i più maligni e i più accaniti ritorsero le loro vendette sull'autore; al quale, perch'era anch'egli, come noi tutti, uomo di carne e di sangue, vollero far parere amara la vita e terribile la vendetta per mezzo della calunnia, della povertà e dell'esilio ${ }^{25}$.

Locke sembra indossare i panni di Jacopo Ortis. La «calunnia», la "povertà», l' «esilio» lo avvicinano a proscritti celebri e gli danno un'aura infelice. Come in una storia esemplare, tuttavia, la grandezza dell'anima resiste alle pressioni del mondo e ne allontana il peso. Simile al più celebre profugo fiorentino di sempre, il Locke che Foscolo esalta utilizza le virtù

23. EN VII, p. 127.

24. In questo senso il filosofo inglese diventa una nuova espressione dei «codici autobiografici» di Foscolo (secondo la formula di S. Gentili, I codici autobiografici di Ugo Foscolo, Roma, Bulzoni, 1997).

25. EN VII, p. I27. 
personali per difendersi da nemici arroganti. Ignora la loro vendetta e si affida completamente al valore del suo lavoro e alla missione che essa contiene:

Che se Giovanni Locke non si fosse armato di onestà, di fortezza e di tutte le virtù che lo studio dell'uomo e la rassegnazione ai decreti della natura possono somministrare ai letterati, s'egli al contrario non avesse cercata che la gloria, e vedendola perseguitata, piagata e derisa, avesse, come pur molti fanno, considerate come sue proprie quelle piaghe e que' vituperi, quest'altissimo ingegno non sarebbe egli stato infelicissimo nel tempo stesso, e non avrebbe egli forse conosciuto che le lettere rivolte all'acquisto della fama o deludono, o affliggono chi le coltiva? ${ }^{26}$

Solo la fiducia nei principi salva l'individuo dalla disperazione. La giustezza della battaglia, combattuta in nome di idee immesse nel centro della vita, protegge da qualunque scoramento. Il Locke, che Foscolo ricostruisce, sembra proiettare l'ombra di un destino condiviso. Le due immagini, la propria e quella del filosofo, si allineano una sull'altra e costruiscono un unico profilo. Grandezza delle idee e arroganza dei nemici sono in relazione simmetrica. L'una sollecita l'altra. Il tema è così decisivo che Foscolo lo riprende poco dopo, sottolineando, in maniera perfino eccessiva rispetto alla reale biografia di Locke, la sequela dei mali attraversati. La gloria, "perseguitata, piagata e derisa", si prolunga nel destino stesso dell'io, "calunniato, perseguitato, esiliato». Le pene subite sono denunciate con un duplice climax ascendente, che potrebbe essere estratto proprio dalle Ultime lettere. In opposizione alla prepotenza degli altri, la forza morale sostiene i grandi uomini e costituisce la loro difesa:

Locke, ch'io vi descrissi ier l'altro perseguitato, calunniato, esiliato, visse nondimeno riposato e soddisfatto nell'animo, perchè, come dic'egli, non gli parea d'avere seguito se non se la verità e la propria coscienza. Così si può dir che l'allontanamento da' propri principj, come l'errare di opinione in opinione e di perplessità in perplessità, è la causa più crudele (?) de' nostri mali ${ }^{27}$.

Nel Ragguaglio l'elogio dello spirito libero, capace di volare sopra la mediocrità degli altri, è ancora più iperbolico: «Ma chiunque studia e fa libri per compiacere all'animo suo vola com'aquila per le solitudini sublimi dell'aria, dove sa di non essere veduta e ammirata ${ }^{28}$.

Il tipo di encomio qui adottato si riconnette ad altri passaggi celeberrimi, modulati sulla stessa dimensione sublime. Analoghe celebrazioni, infatti, adottano il medesimo correlativo simbolico, idoneo a segnare 
la differenza dell'anima nobile dal resto degli uomini e a rivendicare la sua superiorità. Nelle Ultime lettere, la similitudine dell'aquila serve a un'identica funzione («[...] se v'ha taluno nelle cui viscere fremano le generose passioni, o le deve strozzare, o rifuggirsi come le aquile e le fiere magnanime ne' monti inaccessibili») ${ }^{29}$ e, nell'Orazione inaugurale, i paesaggi astrali e remoti, estranei alla vista degli uomini comuni, diventano il regno dell'immaginazione poetica: «[la fantasia] sdegna la terra, vola oltre le dighe dell'oceano, oltre le fiamme del sole, edifica regioni celesti, e vi colloca l'uomo e gli dice: Tu passeggerai sovra le stelle» ${ }^{30}$.

Prioritario, come la legge esclusiva dell'anima, resta l'impegno con la verità. Le parole introduttive del Saggio sull'intelletto possono perfino assurgere, attraverso il filtro della voce dell'Accademico del Ragguaglio, nel manifesto dello spirito libero, che si nutre di meditazione, del lavoro della mente e dell'opera che ne deriva. Egli è devoto solo alle sue idee e al piacere intellettuale che la loro creazione favorisce:

Ricordomi sempre (e sono più anni ch'io non leggo più di que' libri!) della prefazione del libro di Locke. Io, mi pare ch'ei dica, presumendo di dire il vero, disputai di metafisica con gli amici; poi, per convincerli, scrissi fuor della rissa e della acerbità de' pareri una lettera di due pagine; ma volendo persuadere me stesso, le due pagine a poco a poco, in più anni e nelle ore chio non aveva volontà di far altro, crebbero in un grosso volume, e con tanto diletto dell'animo mio, che, se il lettore ne sentirà la metà, non potrà certo pentirsi de' danari e dell'ozio ch'ei vi spendesse. Chi uccella allodole ha tanto piacere quanto chi insegue il cervo e il cinghiale, purchè abbia più amore all'esercizio che al lucro della sua caccia. Or io non mi dorrò mai degli anni e del lavoro, benchè ora forse non mi trovi arricchito di verun frutto. Ma so d'avere esercitato in me L'INTELLETTO: l'anima non ha facoltà nè più sublime di questa, né tale che all'esercizio congiunga maggiore e più perenne soddisfazione $e^{31}$.

Le conquiste di Locke costituiscono una svolta definitiva. Esse avviano una rivelazione che resta un'eredità definitiva: per i contemporanei e per i posteri. Non c'è censura che tenga. La luce ha fugato le tenebre. Si irradia progressivamente raggiungendo altri spiriti, «che con le verità dimostrate da lui illuminarono il nostro ed i secoli che verranno. — ${ }^{32}$. Esperienza

29. EN IV, p. 32I.

30. U. Foscolo, Dell'origine e dell'ufficio della letteratura, cit., p. IO2.

3I. EN VII, p. 252.

32. Ivi, pp. 252-253. Accanto alla verità che illumina la mente Locke offre anche le consolazioni dell'anima: «E desidero ch'Ella sappia ch'io vivo non lieto nè sano, ma vivo a ogni modo, e confortato dalla speranza perch'io "sono certo", come dice S. Paolo, "d'avere una buona coscienza", la quale, benchè falli e pecchi assai volte, non però dissimula a sè medesima i falli e i peccati commessi. E ho letto a questi ultimi giorni un egregio discorso dell'illustre Locke Sul modo d'intendere e interpretare l'Epistole di S. Paolo; ed ho ricavati gran lumi per la mente, ma molta maggiore consolazione per l'anima: V. E. se lo faccia dare dalla biblioteca perchè dev'essere stato sicuramente tradotto in francese» (Ep. IV, pp. 203-204). 
intellettuale, dunque, che è irreversibile: un acquisto della ragione dispiegata; un fondamento di metodo e di sapere, che resta negli annali della storia intellettuale degli uomini.

Come accade nella battaglia per la verità, anche nei fatti della vita Foscolo e Locke sembrano seguire un'identica rotta. La sovrapposizione non riguarda solo il parallelismo dei destini, la somiglianza di traversie che restano, a distanza di luoghi e di spazio, simili. Si estende anche al modo di congedarsi dalla vita. Non è forse solo una curiosità che Foscolo, in una lettera del 22 agosto del I8I2, annunci a Isabella Teotochi Albrizi l'incontro con una biografia di Locke, «mio sacro amico, padre e maestro» e le riferisca la consonanza tra l'epitaffio scritto dal filosofo e quello che egli stesso aveva preparato per sé: «E il mio cuore palpitò di gioia vedendo ch'io benchè in altro modo, aveva fatto cenno de' vizi, dote mia, come di tutti i nipoti d'Eva» ${ }^{33}$.

La somiglianza della vita si completa con la morte. Il «sacro amico, padre e maestro" è assimilato in tutto. Entrambi condividono il rispetto della verità nelle opere e negli atti, la vita in mezzo alle persecuzioni e all'esilio, e perfino la scelta di un'epigrafe, che sia capace di restituire il significato dell'esistenza. Il cerchio è perfettamente saldato. Esiste una genealogia di illustri perseguitati, diventati tali per aver rivelato la verità in funzione dell'interesse comune. A questa famiglia di filantropi, che va da Socrate a Locke e D’Alembert, Foscolo ritiene decisamente di appartenere. Tali sapienti sono i modelli sui cui passi egli procede:

Socrate, Locke, D'Alembert dissero le medesime cose, e patirono più di me! Non ch'io sfidi i pericoli, ma l'uomo d'onore non deve per timore tacere le opinioni utili e vere; e se il sacrificarsi inutilmente è pazzia, il sacrificare il pubblico bene fu sempre viltà ${ }^{34}$.

Le idee sono al servizio del «bene pubblico». Il valore che esse rivestono sta tutto nella grandezza del loro «ufficio».

33. «Finchè abbiate il ritratto del Francese, leggete qui sotto l'epitafio dell'Inglese, e dell'Italo-greco. / Traduzione letterale dal latino. / "Qui presso giace Giovanni Locke: se chiedi chi egli si fosse, ti risponde, ch'ei visse pago della sua mediocrità; educato alle lettere, non le coltivò se non quanto bisognava a sacrificare unicamente alla verità. E ciò imparalo da' suoi scritti, che ti faranno fede assai migliore degli elogi sospetti d'un Epitafio. S'egli ebbe alcune virtù non sono sì grandi ch'ei possa ascriverle a sua lode, nè proporle a te, o passeggero, in esempio. I suoi vizi restino seppelliti col suo cadavere. Che se tu cerchi esempi di costumi, tu li hai nel Vangelo: voglia il cielo che tu non possa trovar altrove esempi di vizi! - Ma l'esempio che tu sei mortale (e ciò ti giovi) lo troverai sovra questo sepolcro, e sovra tutta la terra. - Ch'ei nacque l'anno I632, e che morì l'anno I704 te lo ricordi questa lapide che anch'essa fra non molto dovrà perire”. — / Or vi porrò il mio in latino, come breve. / HUgONis. PHOSCOLI / VITIA. VIRTUS. OSSA / Hic. POST. AN..... / QUiescere. coeperunt. / "Di Ugo Foscolo i vizi la virtù, e le ossa, qui dopo anni.... cominciarono a riposare" " (Ep. IV, p. I08).

34. Ep. III, p. 4I3. 
4. Il legame con Locke non si esaurisce, tuttavia, in questo oroscopo di destini gloriosi e infelici. Esiste un altro piano, più propriamente estetico, che rende ancora più marcato l'influsso del filosofo empirista. Questo aspetto riguarda precisamente la poesia: come tecnica e come espressione, come fusione di idee e di linguaggio. Su tale specifico terreno la dipendenza di Foscolo dal suo interlocutore è dichiarata ed è esibita più volte, e proprio in relazione a un aspetto caratterizzante del linguaggio poetico.

Il rilievo attribuito a Locke come teorico dello stile non è naturalmente una prerogativa solo foscoliana. Basti pensare, in tal senso, all'altissima testimonianza offerta da Cesare Beccaria nella premessa alle Ricerche intorno alla natura dello stile. Il filosofo inglese è esaltato come un battistrada, che ha dischiuso un nuovo modo di dare forma alle idee e di legarle allo stile. Egli fa parte della famiglia di «alcuni sublimi spiriti, che nelle loro opere hanno dato non oscuri cenni di voler connettere lo studio delle belle arti con la nuova maniera di filosofare, e di assoggettarlo all'analisi e al ragionamento». Per Beccaria, Locke "ha incominciato un grande edificio, e i filosofi di questo secolo lo hanno considerabilmente accresciuto e migliorato" ${ }^{35}$.

Foscolo isola un elemento preciso delle considerazioni di Locke e ne fa un tratto organico della sua - per così dire — «filosofia della composizione». Nelle osservazioni Sulla traduzione dell'«Odissea», in un contesto particolarmente delicato per fissare le proprietà di una lingua, Foscolo segnala l'importanza che ha, per l'intelligenza e per la vita della poesia, il capitolo VII del libro III del Saggio sull'intelletto umano, interamente rivolto a esaltare la funzione delle particelle nel testo poetico: «Il capo VII del libro III [del Saggio sull'intelletto umano], ove Giovanni Locke parla delle particelle, racchiude molte profonde ed evidentissime teorie, alle quali i nostri grammatici non hanno pensato mai» ${ }^{36}$.

L'argomento è per Foscolo della massima importanza. Ragionando sulla natura dello stile, basandosi sulle «molte profonde ed evidentissime teorie» del passo di Locke, Foscolo attribuisce alle particelle un ruolo insostituibile nell'economia del discorso poetico. Esse permettono il movimento dei pensieri. Collegano, per differenza o per sviluppo, le parti del ragionamento, imprimendogli l'energia delle idee, generando il loro intreccio, della cui sostanza la tessitura della poesia non può fare a meno.

Questo punto di vista, come si sa bene, aveva legittimato la difesa dei Sepolcri contro Monsieur Guillon ed era stato il nucleo intorno a cui

35. C. Beccaria, Opere, a cura di S. Romagnoli, Firenze, Sansoni, I97I, p. 203.

36. EN VII, p. 206. 
si costituiva un'idea di lirica sublime. In modo ancora più asseverativo, Foscolo ribadisce l'importanza della teoria lockiana in una lettera del 27 agosto I8I2. Contestando l'autorità della Crusca e la sua legittimità a indossare i panni di custode del tesoro della lingua italiana, egli rinnova la centralità necessaria delle particelle nella trama del discorso poetico e assegna per l'ennesima volta il merito di questa scoperta alla riflessione di Locke:

Ma dove la Crusca pecca imperdonabilmente si è nelle particelle, le quali in ogni idioma sono le vere e sole giunture delle idee principali del discorso; danno inoltre $i$ toni e mezzitoni come nella musica; ed aiutano lo scrittore a quel chiaroscuro che tanto è più grato quanto le minime tinte che lo distinguono spiccano meno. - Segno questi versi perch'io (non so se meritamente) mi fo bello di questa definizione delle particelle; del resto il profondissimo Locke nell'opera sua maggiore ne parla con altri termini, ma con lo stesso principio, e con quella eloquenza sicura, calda, e tranquilla ad un tempo che nessun filosofo ha mai conseguito ${ }^{37}$.

Foscolo, nelle osservazioni sulla traduzione dell'Odissea, riflette in particolare sulle potenzialità della particella «ma»: "congiunzione sordida, evasiva, bastarda e cavillosa», come la definisce un personaggio di Scott nell' Antiquario. Proprio a questa particella Locke, nel citato capito VII del libro III, aveva dedicato osservazioni preziose, che trovano un'eco prolungata nella riflessione foscoliana. Per il filosofo «Mais est une des particules les plus communes», e «cependant il me semble qu'elle donne à entendre divers rapports que l'Esprit attribüe à différentes Propositions ou parties de Propositions qu'il joint par ce Monosyllabe. [...] Prémiérement, cette Particule sert à marquer contrariété, exception, différence». Inoltre, "elle sert à rendre raison de quelque chose dont on se veut excuser. [...] ${ }^{38}$; oppure, in altro modo, "sert quelquefois de transition». Locke aggiunge che potrebbe indicare ancora altre sfumature nell'uso della particella. Il suo scopo, tuttavia, non implica la volontà di catalogare tutti i casi possibili. Gli esempi proposti servono a un altro fine. Essi «pourront donner occasion de réfléchir sur l'usage et la force que ces Mots ont dans le discours».

Foscolo accetta i presupposti dei «divers rapports» generati dalle congiunzioni nell'architettura del testo e se ne serve per definire un tratto necessario della tessitura poetica. Le «molte minime idee accessorie e concomitanti, che danno sempre più movimento e più tinte al significato

37. Ep. IV, p. II6.

38. J. Locke, Essai philosophique concernant l'entendement humain, Amsterdam, Pierre Mortier, I735, p. 38I. Foscolo leggeva Locke in questa traduzione. La traduzione francese di Euvres diverses di Locke è presente tra i libri posseduti da Foscolo. Cfr. G. Nicoletti (a cura di), La biblioteca fiorentina del Foscolo nella Biblioteca Marucelliana, Firenze, Biblioteca Marucelliana, 1978. Cfr., in questo volume, p. 3I, n. 65. 
primitivo» di una parola, sono suggerite precisamente dall'azione delle particelle. Tra loro, Foscolo, a titolo di esempio, allega precisamente il «ma»: «basta che chiunque scrive consideri i diversi accidenti della particella $m a{ }^{39}$. Le congiunzioni si offrono come generatrici di moto, il quale, insieme con l'armonia e il colorito, costituisce uno dei caratteri dello stile. Sono, perciò, un fattore fondamentale della sua ricchezza ed efficacia.

L'analisi del linguaggio, avviata dal filosofo inglese, diventa un modo per ragionare sulle connessioni che si stabiliscono tra i pensieri e le parole. Nelle Epoche Foscolo così proclama: «Locke [...] facilitò lo studio delle analisi delle idee, e quindi della natura delle lingue, e Condillac [...] illustrò questa difficilissima parte della metafisica» ${ }^{40}$. Nello stesso tempo, il primato della sensazione ${ }^{4 \mathrm{I}}$ nell'esperienza degli uomini, destinatari della poesia, impone che l'atto poetico produca sul lettore l'effetto cercato. Il linguaggio della poesia dà un corpo all'astrazione del pensiero e, nello stesso tempo, attraverso la forma che assume, stimola le passioni del lettore. Le conquiste del sensismo sono in questo campo, per Foscolo, un punto d'arrivo. Perché la parola sia efficace, deve agire sulla mente attraverso i sensi. Se questo processo non si compie, la parola resta sterile e inerte.

Non stupisce, perciò, che, più volte, Foscolo richiami l'obbligo di mettere in movimento i sensi del lettore. Fino alla Dissertation del I822, teorizza che «ogni allegoria è, in effetti, solo la personificazione di un'idea astratta, che, agendo più rapidamente e agevolmente sui nostri sensi e sulla nostra fantasia, conquista con più rapidità l'intelletto» ${ }^{42}$. Solo i critici metafisici possono ignorare il valore che le allegorie hanno avuto, giacché esse "costituirono i materiali più eleganti e ricchi di spunti per il lavoro dell'artista».

39. EN VII, p. 206.

40. EN XI/I, p. I56.

4I. Quanto questo primato pesi anche nella dinamica della psicologia individuale è documentato dal passo che segue: «Ma sopra queste materie ad tuum Apollinem referendum censeo. - A pagina 50-52 mi sono accorto ch'Ella intendeva di parlare di me; e le Sono gratissimo, dacchè conosco che nè la meditazione di soggetti importanti, nè la cura dell'eloquenza hanno potuto torle le rimembranze ch'io bramo e serbo perpetue, e delle quali mi compiacerò sempre. Il detto di Medea in Ovidio: Video meliora proboque, deteriora sequor, è profondamente sviscerato da Giovanni Locke nel suo trattato dell'intendimento. Non mi ricordo del luogo; ma so ch'egli ne parla a lungo, e prova che gli uomini sono perpetuamente e necessariamente mossi dalla più forte sensazione, e che si opera il male presente ad onta delle ragioni poste innanzi dalla esperienza del passato, e dalle previdenze del futuro pel solo motivo che le cose presenti fanno più forza all'animo nostro. S'Ella, signor Conte, avesse la versione del Coste, potrà facilmente trovare quel ragionamento del filosofo inglese; non lo troverà ne' compendi e nelle versioni italiane, perchè s'è avuto cura di troncare ogni sentenza ed ogni argomento che ferisse la religione» (Ep. III, pp. 538-539).

42. EN I, p. IO97. 
All'interno di un quadro teorico così delineato, Locke arriva a intrecciarsi con Bacone e con Vico. Essi indicano, con lo stesso vigore, la produttività conoscitiva delle favole. Gli antichi miti rappresentano idee; contengono valori; drammatizzano vizi e virtù. Attraverso la concretezza dei loro racconti, mostrano agli uomini «la sapienza morale e politica dei primi filosofi» ${ }^{43}$. Perciò si propongono come la grammatica stessa della poesia. Ne costituiscono l'anima necessaria, giacché custodiscono, nei loro involucri, la sapienza dei popoli e la memoria della loro humanitas. Solo "le idee» che non si arrendono "ai sensi, rifuggono dall'intelletto» ${ }^{44}$.

Come Foscolo scrive nell' Orazione, fondendo in un solo ragionamento Locke e Condillac, accade che «quanto più i sensi s'invigoriscono alle impressioni, e le interne potenze si esercitano a concepire, gli organi della parola si vanno più distintamente snodando» ${ }^{45}$.

Ed è opportuno aggiungere, sulla scorta delle indicazioni di Enzo Neppi, che «Foscolo usa argomenti di provenienza diversa, proprio perché è convinto della complessità dei fenomeni e vuole sottrarsi alle due alternative fondamentali elaborate dal pensiero politico del Settecento e dell'Ottocento: ritorno all'origine $[\ldots]$ oppure progresso» ${ }^{46}$.

A questo punto si può concludere, sostenendo, come avverte Andrea Battistini, che «elementi vichiani vivano in simbiosi con motivi che in origine furono di Machiavelli, di Hobbes, di Locke, con la stessa antropologia vichiana mediata dagli esuli napoletani e magari da un'elaborazione personale dello stesso Foscolo» ${ }^{47}$ : autori tenuti insieme e fusi in un'ideologia "connotata dall'alta densità di coagulo» ${ }^{48}$ di una biblioteca affollata ed eterogenea. Prende forma, in questa costellazione di autori e d'idee, un grandioso tentativo di elaborare un'arte antica e classica. Il sensismo e l'antropologia poetica vichiana autorizzano e legittimano l'uso della mitologia; le idee "concomitanti e accessorie» contrassegnano una lirica di specie sublime,

43. U. Foscolo, Dell'origine e dell'ufficio della letteratura, cit., p. I25.

44. Ivi, p. I23.

45. "Ogni uomo sa che la parola è mezzo di rappresentare il pensiero; ma pochi si accorgono che la progressione, l'abbondanza e l'economia del pensiero sono effetti della parola. E questa facoltà di articolare la voce, applicandone i suoni agli oggetti, è ingenita in noi e contemporanea alla formazione de' sensi esterni e delle potenze mentali, e quindi anteriore alle idee acquistate da' sensi e raccolte dalla mente; onde quanto più i sensi s'invigoriscono alle impressioni, e le interne potenze si esercitano a concepire, tanto gli organi della parola si vanno più distintamente snodando" (Ivi, p. 99).

46. E. Neppi, Foscolo pensatore europeo, cit., p. 73.

47. A. Battistini, Temi vichiani nei «Sepolcri», in F. Danelon (a cura di), «A egregie cose». Studi sui «Sepolcri» di Ugo Foscolo", Venezia, Marsilio, 2008, p. 32.

48. G. Mazzacurati, Retaggi vichiani nella filologia e nella storiografia del Foscolo, in M. Santoro (a cura di), Foscolo e la cultura meridionale, Napoli, SEN, I980, pp. 45-46. 
come quella dei poeti sacerdoti di un tempo remoto. La rivoluzione di Locke riporta Foscolo nel cuore del classicismo e delle sue ragioni.

Antico e nuovo si ricongiungono, si mescolano, si combinano l'uno con l'altro. L'emozione dei vecchi poemi si ripete nella forma di uno stile eletto, che proprio dalla loro maniera ricava la sua esistenza: «Ho desunto questo modo di poesia da' Greci, i quali dalle antiche tradizioni traevano sentenze morali e politiche presentandole non al sillogismo de' lettori, ma alla fantasia ed al cuore».

D’altra parte, proprio Vincenzo Cuoco, costruendo la genealogia degli autori della sua epoca, allestiva un canone composito, in cui Locke, Du Marsais, Condillac, Beccaria e Vico potevano coesistere come aspetti di una sola, grandiosa impresa ${ }^{49}$ : la costruzione delle scienze umane all'alba del secolo XIX.

49. «Nell'epoca in cui Locke scopriva per le vie della ragione, rapporti tra le idee e le parole, quei rapporti che, sviluppati e promossi da Condillac, Du Marsais, e da Beccaria, ci avrebbero dovuto dare una grammatica ed una rettorica nuova e degna di Aristotele e di Platone, nella stessa epoca Vico per la via de' fatti applicava lo studio delle lingue alla storia delle nazioni, ed all'analisi della mente del genere umano" (V. Cuoco, Pagine giornalistiche, a cura di F. Tessitore, Bari, Laterza, 20II, pp. I09-IIo). 